\title{
Síndrome de Rapunzel. Reporte de un caso
}

\author{
Vanessa Durán Becerra, ${ }^{1}$ Laura Tatiana Padilla Pinzón, ${ }^{1}$ Jean Andre Pulido Segura, ${ }^{1}$ Luis Felipe \\ Cabrera Vargas, ${ }^{1}$ Mauricio Pedraza Ciro, ${ }^{1}$ Sebastián Sánchez Ussa, ${ }^{1}$ Erick Vinck Geerman, ${ }^{1}$ \\ Tomás Arce ${ }^{2}$
}

${ }^{1}$ Departamento de Medicina y Cirugia General, Universidad El Bosque.
${ }^{2}$ Departamento de Medicina, Universidad de Ciencias Aplicadas y Ambientales.
Bogotá, Colombia.

Acta Gastroenterol Latinoam 2020;50(3):341-344

Recibido: 20/03/2019 / Aceptado: 15/05/2019 / Publicado online: 28/09/2020

\section{Resumen}

Los bezoares son acúmulos de material no digerible dentro del tracto gastrointestinal clasificados según el material que los conforma. Se denominan tricobezoares a aquellos bezoares formados por el cabello, que al extenderse delante de la cámara gástrica toman el nombre de sindrome de Rapunzel. Se presentan principalmente en la adolescencia asociados a trastornos psiquiátricos, tienen manifestaciones clínicas variadas y la endoscopia de vías digestivas altas es el estándar de oro para su diagnóstico. Presentamos el reporte de un caso de sindrome de Rapunzel en una paciente de 18 años con dolor abdominal, emesis y ausencia de deposiciones. Desde el punto de vista clínico, se evidenció la obstrucción intestinal por lo que se decidió realizarle una laparotomía exploratoria donde se encontró un tricobezoar que se extendía adelante del piloro. Según la literatura, la cirugía abierta es el procedimiento más exitoso para la resolución de esta enfermedad.

Palabras claves. Bezoar, endoscopia, laparotomía.

Correspondencia: Jean Pulido Segura Grupo de investigación en Cirugia General y Subespecialidades. Universidad El Bosque. Bogotá, Colombia

Tel.: 00573112726157

Correo electrónico: japulido@unbosque.edu.co

\section{Rapunzel Syndrome. Case report}

\section{Summary}

Bezoars are accumulations of non-digestible material in the gastrointestinal tract; classified according to the material that it is composed. They are called trichobezoars to those who are formed by the hair, and when are extended to the gastric chamber are called Rapunzel syndrome. It presents mainly in teenagers associated with psychiatric disorders. It has different clinical manifestations and the upper gastrointestinal endoscopy is the gold standard for its diagnosis. We present a case of a Rapunzel Syndrome in an 18 years old patient who was admitted due to abdominal pain, emesis and absence of stools. The initial diagnosis was a difficult diagnosis because of an intestinal obstruction. By this reason it was decided to perform an exploratory laparotomy where it was found a trichobezoar which was extended over the pylorus. According to literature, Laparotomy is the most successful procedure for the resolution of this disease.

Key words. Bezoars, endoscopy, laparotomy.

\author{
Abreviaturas \\ TGI: Tracto gastrointestinal. \\ VEDA: Videoendoscopia digestiva alta. \\ TRB: Tricobezoares. \\ SR: Sindrome de Rapunzel. \\ TAC: Tomografia axial computarizada. \\ BR: Bezoares
}




\section{Introducción}

Los bezoares son acúmulos de material no digerible a nivel del tracto gastrointestinal (TGI) con un efecto de masa, clasificados según el material que los conforma. Pueden estar constituidos por materiales sintéticos, como el plástico, hasta biológicos, como el pelo, llamados tricobezoares (TRB). Cuando estos últimos se extienden por delante de la cámara gástrica toman el nombre de síndrome de Rapunzel (SR). Presenta manifestaciones clínicas inespecíficas, por lo que se lo considera un reto diagnóstico. El grado de afectación incluye desde casos asintomáticos hasta pacientes con obstrucción intestinal y sepsis por perforación de la víscera hueca generando compromiso sistémico. Por esto, es importante reconocer la presentación clínica aguda y los factores predisponentes como los trastornos psiquiátricos. ${ }^{1,2}$

Por lo anterior, presentamos el caso de una paciente de 18 años. Analizamos aspectos clínicos y quirúrgicos del manejo del SR. Además, realizamos una revisión de la literatura teniendo en cuenta estudios publicados en la base de datos PubMed, en los últimos 5 años, en humanos, en los idiomas inglés y español. Detectamos 148 casos (Tabla 1).

Tabla 1. Casos de bezoares reportados en los últimos 5 años en PUBMED

\begin{tabular}{lcc}
\hline & $\begin{array}{c}\text { Número } \\
\text { de casos }\end{array}$ & Porcentaje (\%) \\
Casos reportados de Bezoares & 148 & 100 \\
Tricobezoares & 53 & 35,81 \\
$\begin{array}{l}\text { Fitobezoares (incluido plástico } \\
\text { bezoar, lactobezoar y metal bezoar) }\end{array}$ & 51 & 34,45 \\
Fármaco bezoares & 44 & 29,72 \\
\hline
\end{tabular}

\section{Caso clínico}

Presentamos el caso de una paciente de 18 años, quien ingresó a urgencias por un cuadro clínico de tres días de dolor tipo cólico en epigastrio, no irradiado, de intensidad 8/10 en escala subjetiva del dolor, asociado al emesis y a la ausencia de deposiciones de cuatro días de evolución. En el examen físico el abdomen fue blando, sin hallazgos de masa, doloroso en epigastrio sin signos de irritación peritoneal. Se tomaron exámenes paraclínicos que mostraron una leucocitosis de predominio neutrofílico, sin anemia ni alteraciones electrolíticas. Se le realizó una videoendoscopía digestiva alta (VEDA) por la persistencia del dolor y por la sospecha inicial de una enfermedad ácido péptica. La VEDA reportó una masa redondeada a nivel del antro, de color verdoso que no se dejó desplazar, constituida por cabellos que impedían la valoración de estructuras anatómicas posteriores, concordante con TRB. Finalmente se le realizó una laparotomía exploratoria que mostró una masa, que ocupaba toda la cámara gástrica, que se extrajo sin complicaciones (Figura 1). Tras 48 horas se probó la tolerancia a la vía oral y se le realizó una reposición periférica del potasio por hipokalemia, como única complicación.

Figura 1. Tricobezoar encontrado en la paciente

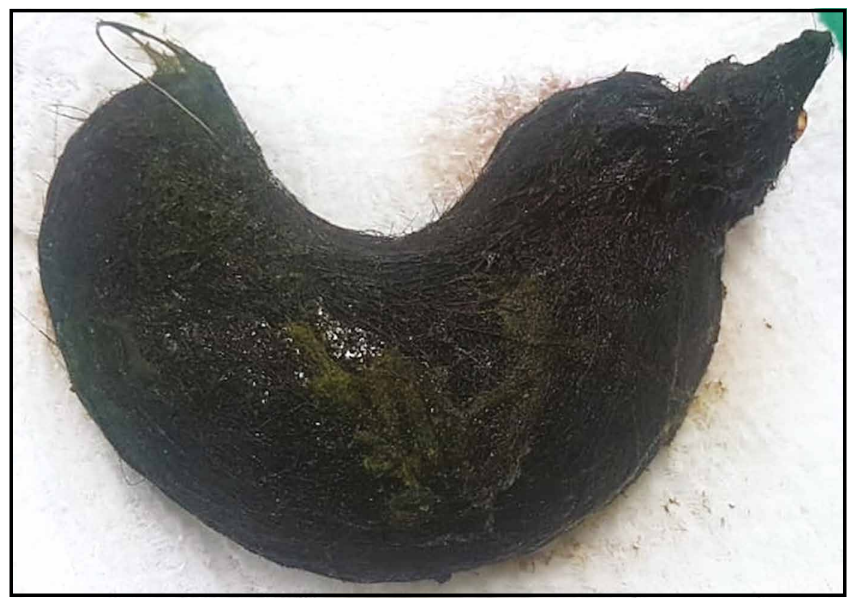

La paciente fue valorada por psiquiatría, quienes reportaron ideas de minusvalía, soledad, muerte y suicidio por violencia intrafamiliar, además de antecedentes de tricotilomanía y tricofagía referidos por los familiares. La paciente fue dada de alta sin complicaciones referidas al procedimiento.

\section{Discusión}

Los bezoares (BR) son clasificados según el material que los conforma en fitobezoares (materiales orgánicos no digeribles), lactobezoares (productos lácteos), fármaco bezoares (medicamentos) y TRB (cabello). Estos últimos, al extenderse delante de la cámara gástrica, toman el nombre de síndrome de Rapunzel. ${ }^{1,3,4}$

Se obtuvieron 148 casos de la base de datos PubMed correspondientes a BR. El 35,81\% era TRB, una patología rara e infrecuente asociada a trastornos psiquiátricos como la tricotilomanía, la tricofagía y los trastornos de ansiedad, afectando principalmente a las mujeres jóvenes. Bargas et al. calculan una razón de 10:1 mujeres a hombres, similar a nuestra revisión, en la que el 98,1\% de los 
pacientes es de sexo femenino y el único caso masculino se relacionó con un paciente que consumía hilos de tela. ${ }^{2}$ Es una enfermedad ligada a la adolescencia y a la adultez temprana dado que los factores de riesgo para enfermedades psiquiátricas se desarrollan en esta etapa. Se estima que entre el 80 y $94 \%$ de los pacientes son menores de 30 años (Tabla 2). 4,5

Tabla 2. Distribución demográfica de casos de tricobezoares

\begin{tabular}{|c|c|c|}
\hline & $\begin{array}{c}\text { Número } \\
\text { de pacientes }\end{array}$ & Porcentaje (\%) \\
\hline Casos de TRB & 53 & 35,81 \\
\hline Rango de edad & \multicolumn{2}{|c|}{2 a 69 años } \\
\hline TRB en menores de 18 años & 43 & 81,13 \\
\hline TRB en menores de 30 años & 50 & 94,34 \\
\hline Mujeres con TRB & 52 & 98,11 \\
\hline Hombres con TRB & 1 & 1,89 \\
\hline Casos con SR & 19 & 35,85 \\
\hline \multicolumn{3}{|l|}{ Resolución con: } \\
\hline - laparotomía + gastrostomía & 46 & 86,79 \\
\hline - laparotomía + gastrostomía directa & 37 & 69,81 \\
\hline $\begin{array}{l}\text { - laparotomía + gastrostomía post } \\
\text { endoscopía fallida }\end{array}$ & 9 & 16,98 \\
\hline Laparoscopia & 1 & 1,89 \\
\hline Resolución por endoscopía & 6 & 11,32 \\
\hline Paciente con anemia al ingreso & 11 & 20,75 \\
\hline Trastornos psiquiátricos asociados & 47 & 88,68 \\
\hline
\end{tabular}

TRB: tricobezoar; SR: síndrome de Rapunzel.

Los otros bezoares se encuentran principalmente en los adultos mayores, con factores de riesgo como las enfermedades gástricas, que generan alteración de la mucosa y estados posquirúrgicos abdominales que reducen la motilidad y retrasan el vaciamiento gástrico. La gastroparesia diabética, la infiltración del nervio vago secundaria a in- vasión tumoral o cualquier patología o medicamento que altere la función gastrointestinal también son condiciones predisponentes. ${ }^{13}$ Pueden presentarse de manera asintomática, como un hallazgo, o con manifestaciones clínicas variadas. Las manifestaciones más frecuentes incluyen la masa palpable en el epigastrio $(70 \%)$, la pérdida de peso (38\%), el dolor abdominal (37\%), las náuseas y los vómitos $(33 \%)$, la diarrea y la anorexia (32\%), así como la sensación de plenitud y la anemia por la deficiencia de hierro (asociada a pica como un factor desencadenante del SR). En nuestra revisión de la literatura, sólo el 20,75\% de los 53 casos reporta una asociación con la anemia. El resultado de esta masa ocupante de la cámara gástrica puede generar úlceras gástricas por presión siendo la causa de los síndromes anémicos. La obstrucción intestinal asociada a la perforación es una de las manifestaciones más graves. ${ }^{1,3,4,6}$

El SR compromete el TGI y el extremo distal del tricobezoar se extiende hasta el duodeno, el íleon, el colon derecho o incluso hasta el recto. Allí se adhiere a los pliegues de la mucosa gástrica y es retenido por su pobre superficie de fricción necesaria para la propulsión, generando una obstrucción o seudo-obstrucción intestinal, convirtiéndose en una urgencia quirúrgica. ${ }^{1,4,7}$

El enfoque hacia el paciente dependerá del estado al momento de su identificación y el éxito variará según el tamaño de la masa. Nuestra revisión evidencia que a mayor tamaño y consistencia del TRB hay más asociación con el tratamiento endoscópico fallido (Tabla 2). Para los fitobezoares, por ejemplo, se han reservado los manejos conservadores, como la disolución de la masa por sustancias químicas (bebidas cola) o papaína. Sin embargo, el manejo de TRB suele ser más invasivo dado que no es posible la disolución química y frecuentemente requieren la intervención quirúrgica, como en el caso de nuestra paciente. ., 7-11 $^{2}$

Belsky et al. informan una sensibilidad de las radiografías abdominales para los bezoares de menos del 18\%, siendo la tomografía axial computarizada más confiable, pues muestra una masa circunscrita en la región gástrica, compuesta de espirales concéntricas de diferentes densidades con burbujas enredadas dentro. ${ }^{3}$ Sin embargo, la endoscopia es la herramienta diagnóstica más conveniente dado que permite estimar el tamaño y la ubicación a través de la visión directa de la masa e incluso su extracción. Debido a la aparición tardía de los síntomas, la masa suele ser muy compacta y superar los $20 \mathrm{~cm}$ de diámetro, lo que impide su extracción por la vía endoscópica y requiere una intervención quirúrgica, como muestra nuestra paciente. Los casos publicados exhiben que el 86,79\% requirió una intervención quirúrgica y el 16,98\% de estos fue debido a una endoscopia fallida. . $^{6,10,12}$ 


\section{Conclusiones}

El síndrome de Rapunzel es una entidad poco frecuente presentada generalmente en las mujeres con trastornos psiquiátricos. Es importante conocer esta patología y su amplio espectro de presentaciones clínicas dada su prevalencia en las personas con patologías psiquiátricas. Si bien la endoscopía representa la mejor herramienta para el diagnóstico, su uso suele ser muy limitado para la extracción, en función del tamaño y la consistencia, requiriendo generalmente de la cirugía para su resolución.

Agradecimientos. Al departamento de Cirugía General de la Universidad El Bosque, a su grupo de investigación de Cirugía General y Subespecialidades y al Hospital San Rafael de Fusagasugá por su apoyo en los proyectos de investigación, así como el aporte de la historia clínica y documentos brindados.

Sostén Financiero. Los autores declaramos no contar con financiamiento de terceros.

\section{Referencias}

1. Islek A, Sayar E, Yilmaz A, Boneval C, Artan R. A rare outcome of iron deficiency and pica: Rapunzel syndrome in a 5-year-old child iron deficiency and pica. Turk J Gastroenterol 2014; 25 (1): 100-102.

2. Bargas Ochoa M, Xacur Hernández M, Espadas Torres M, Quintana Gamboa A, Tappan Lavadores I, Méndez Domínguez N. Síndrome de Rapunzel con doble tricobezoar simultáneo en una adolescente: Reporte de caso. Revista chilena de pediatría 2018; 89 (1): 98-102.
3. Belsky J, Whitcomb V, Zimmerman E, Stankovic C. Rapunzel Syndrome: diagnosis via radiograph and history. Pediatric Emergency Care 2014; 30 (5): 352-353.

4. Germani M, Beltrà Picó R, Hernández Castelló C. Síndrome de Rapunzel: tratamiento laparoscópico. Anales pediatría (Barc) 2014; 80: e33-34.

5. Bousfiha N, Derieux S, Amiot A, Cherif R. Upper gastrointestinal obstruction due to trichobezoar. Presse Med 2014; 43 (9): 1008-1009.

6. Caiazzo P, Di Lascio P, Crocoli A, Del Prete I. The Rapunzel syndrome. Report of a case. G Chir 2016; 37 (2): 90-94.

7. Mariotto A, Peretti M, Scirè G, Mantovani A, Zambaldo S, Pani E, Saverio Camoglio F, Giacomello L. Trichobezoars in children: therapeutic complications. La Pediatria Medica e Chirurgica 2014; 36 (5-6): 101.

8. Aoi S, Kimura K, Tsuda T. Double and synchronous trichobezoars causing small-bowel obstruction and detected by multidetector computed tomography: report of two cases. Surgery Today 2015; 45 (5): 634-637.

9. Cook SL, Beaver B, Brislin R, Elitsur Y. Rapunzel syndrome: not just a "hairy tail". Clinical Pediatrics (Phila) 2011; 50 (4): 372-374.

10. Beristain-Silva JL, Cordero Barberena R. Beristain Hernández JL. Síndrome de Rapunzel: una causa rara de dolor abdominal. Revista de gastroenterología de México 2016; 81 (3): 178-179.

11. Irastorza I, Tutau C, Vitoria JC. A trichobezoar in a child with undiagnosed celiac disease: a case report. World J Gastroenterol 2014; 20 (5): 1357-1360.

12. Nour I, Abd Alatef M, Megahed A, Yahia S, Wahba Y, Shabaan AE. Rapunzel syndrome (gastric trichobezoar), a rare presentation with generalised oedema: case report and review of the literature. Paediatrics and International Child Health 2019; 39 (1): 76-78. 